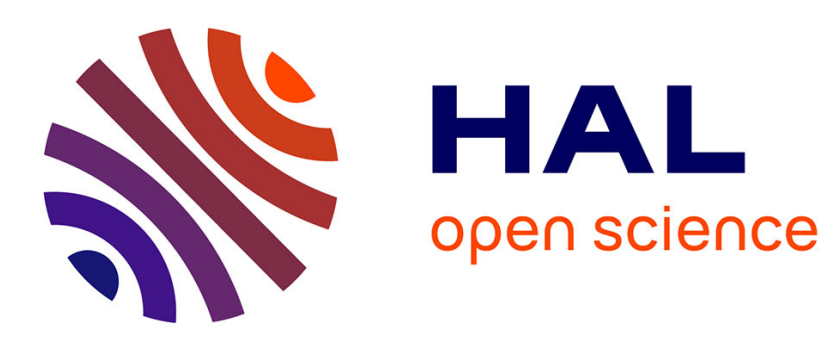

\title{
Réduction de modèle par identification de modes dominants : application à un modèle bidimensionnel de diffusion thermique
}

D. Petit, R. Pasquetti

\section{- To cite this version:}

D. Petit, R. Pasquetti. Réduction de modèle par identification de modes dominants: application à un modèle bidimensionnel de diffusion thermique. Revue de Physique Appliquée, 1990, 25 (8), pp.831-842. 10.1051/rphysap:01990002508083100 . jpa-00246245

HAL Id: jpa-00246245

https://hal.science/jpa-00246245

Submitted on 1 Jan 1990

HAL is a multi-disciplinary open access archive for the deposit and dissemination of scientific research documents, whether they are published or not. The documents may come from teaching and research institutions in France or abroad, or from public or private research centers.
L'archive ouverte pluridisciplinaire HAL, est destinée au dépôt et à la diffusion de documents scientifiques de niveau recherche, publiés ou non, émanant des établissements d'enseignement et de recherche français ou étrangers, des laboratoires publics ou privés. 


\title{
Réduction de modèle par identification de modes dominants : application à un modèle bidimensionnel de diffusion thermique
}

\author{
D. Petit ( $\left({ }^{1}\right)$ et R. Pasquetti ( $\left.{ }^{2}\right)$ \\ (') Laboratoire Systèmes Energétiques et Transferts Thermiques, URA CNRS 1168, Centre de St Jérôme, \\ Avenue Escadrille Normandie-Niemen, 13397 Marseille Cedex 13, France \\ ( ${ }^{2}$ Département de Mathématiques, URA CNRS 168, Faculté des Sciences Valrose, 06034 Nice Cedex, \\ France
}

(Reçu le 20 février 1990, accepté le 15 mai 1990)

\begin{abstract}
Résumé. - Dans le cadre de la modélisation des systèmes dynamiques linéaires, on présente différentes formulations des équations d'état et on analyse leur intérêt quant au problème de la réduction de modèle. On montre ensuite comment, à partir d'un modèle de connaissance, un modèle réduit peut être obtenu par une méthode originale d'identification de ses modes dominants. Cette méthode est appliquée à un modèle bidimensionnel (d'ordre 150), décrivant la diffusion thermique au sein d'un plancher chauffant, et comparée à d'autres techniques de réduction (Eitelberg, Marshall et agrégation). Les simulations numériques montrent que le modèle identifié (d'ordre 4 ) donne des résultats très satisfaisants.
\end{abstract}

\begin{abstract}
In the framework of the dynamic linear models, several forms of the state space representation are pointed out. An analysis of the interest of these forms for model reduction is presented. Then, it is shown how to obtain a low order model from a large one, by using an original method of identification of preponderant modes. This technique is applied to a bidimensional model (of order 150), describing the diffusive heat transfert across a heating floor. Numerical simulations attest that the identified model (of order 4) is quite satisfying. This obtained model is also compared to other reduced models coming from other methods (Eitelberg, Marshall and aggregation).
\end{abstract}

\section{Nomenclature.}

\section{Matrices:}

$\boldsymbol{A}$ : matrice d'évolution (dim. $n, n)$

A : matrice d'évolution du modèle réduit $(\operatorname{dim} . m, m)$

$\boldsymbol{B}$ : matrice de commande $(\operatorname{dim} . n, p)$

B : matrice de commande du modèle réduit (dim. $m, p$ )

C : matrice d'observation (dim. $q, p)$

C : matrice d'observation de l'état réduit (dim. $q, p)$

$\boldsymbol{C}_{\mathrm{a}}$ : matrice diagonale des capacités thermiques (dim. $n, n)$

D : matrice de couplage entrées - sorties $(\operatorname{dim} . q, p)$

$\boldsymbol{G}$ : matrice des conductances thermiques (dim. $n, n)$

$\boldsymbol{M}$ : matrice modale (dim. $n, n)$

$\boldsymbol{\Gamma}$ : matrice de commande dans la base modale $(\operatorname{dim} . n, p)$
$\Gamma$ : matrice de commande du modèle réduit dans la base modale (dim. $m, p$ )

$\boldsymbol{\Lambda}$ : matrice diagonale des valeurs propres de $\boldsymbol{A}$ (dim. $n, n)$

$\boldsymbol{\Lambda}$ : matrice diagonale des valeurs propres de $\mathbf{A}$ (dim. $m, m$ )

$\boldsymbol{\Omega}$ : matrice d'observation dans la base modale $(\operatorname{dim} . q, n)$

$\mathbf{\Omega}$ : matrice d'observation du modèle réduit dans la base modale (dim. $q, m$ )

Vecteurs :

$T$ : vecteur des températures $(\operatorname{dim} . n)$

$u$ : vecteur des entrées $(\operatorname{dim} . p)$

$x$ : vecteur d'état $(\operatorname{dim} . n)$

$\mathrm{x}$ : vecteur d'état réduit $(\operatorname{dim} . m)$

$y$ : vecteur des sorties $(\operatorname{dim} . q)$

$\hat{y}$ : estimation du vecteur des sorties $(\operatorname{dim} . q)$

$\xi$ : vecteur d'état dans la base modale (dim. $n$ )

$\xi$ : vecteur d'état du modèle réduit dans la base modale (dim. $m$ ) 
Scalaires :

$t$ : temps

$c$ : capacité thermique massique

$k$ : conductivité thermique

$\lambda$ : valeur propre de $\boldsymbol{A}$

$\lambda$ : valeur propre de $\mathbf{A}$

$\rho$ : masse volumique

Sigles :

MC : Modèle Complet

MI : Modèle Identifié (réduit)

Remarque : les notations en italiques correspondent aux modèles complets et les notations en caractères droits aux modèles réduits.

\section{Introduction.}

La modélisation de systèmes thermiques régis par des équations aux dérivées partielles nécessite en général une discrétisation spatiale et les méthodes numériques habituellement utilisées (éléments finis, différences finies, méthode nodale, etc...) conduisent alors à une représentation par un système de $n$ équations différentielles du premier ordre en temps $t$ :

$$
\boldsymbol{C}_{\mathrm{a}} \dot{T}(t)=\boldsymbol{G} T(t)+\boldsymbol{\Phi}(t)
$$

$T(\operatorname{dim} . n)$ est le vecteur des températures aux $n$ nœuds de la discrétisation et $\dot{T}$ est sa dérivée temporelle. Dans le cas des systèmes linéaires invariants, les matrices (dim. $n, n) \boldsymbol{C}_{\mathrm{a}}$ et $\boldsymbol{G}$ sont constantes. Pour la méthode nodale, qui sera utilisée dans la suite de ce travail, $\boldsymbol{C}_{\mathrm{a}}$ est la matrice diagonale des capacités thermiques, $\boldsymbol{G}$ la matrice des conductances thermiques entre nœuds voisins, $\Phi$ le vecteur des flux associés aux conditions limites et aux sources de chaleur.

Cette formulation se prête bien à la mise sous forme des équations d'état classiquement utilisées en réduction de modèle :

$$
\begin{aligned}
& \dot{x}(t)=\boldsymbol{A} x(t)+\boldsymbol{B} u(t) \\
& y(t)=\boldsymbol{C} x(t)
\end{aligned}
$$

où $x=T$ est le vecteur d'état $(\operatorname{dim} . n), u(t)$ est le vecteur des entrées dont les composantes représentent les termes sources ou les conditions limites, $y$ est le vecteur des sorties (dim. $q$ ) où sont groupées les températures ou flux dont on veut suivre l'évolution temporelle. $\boldsymbol{A}, \boldsymbol{B}, \boldsymbol{C}$ de dimensions appropriées, sont respectivement les matrices d'évolution, d'entrée et d'observation. Les relations (1) et (2.1) donnent immédiatement :

$$
\boldsymbol{A}=\boldsymbol{C}_{\mathrm{a}}^{-1} \boldsymbol{G} \quad \boldsymbol{B} u(t)=\boldsymbol{C}_{\mathrm{a}}^{-1} \boldsymbol{\Phi}(t) .
$$

La relation (2.1) prend une forme particulièrement intéressante lorsque la matrice d'évolution $\boldsymbol{A}$ est diagonalisable. En effet, de nombreux travaux, tant d'automaticiens [1-5] que de thermiciens [6-10] ont mis en avant l'intérêt de la représentation (2) quand elle est exprimée dans la base des vecteurs propres. Si on suppose effectivement l'existence de $n$ valeurs propres distinctes pour $\boldsymbol{A},(2)$ peut alors s'écrire :

$$
\begin{aligned}
& \dot{\xi}=\Lambda \xi+\Gamma u \\
& y=\Omega \xi
\end{aligned}
$$

où $\xi$ est le nouveau vecteur d'état, $\Lambda$ la matrice diagonale des valeurs propres, $\Gamma$ et $\Omega$ les nouvelles matrices de commande et d'observation telles que :

$$
\begin{aligned}
\xi=M^{-1} x \quad \Gamma=M^{-1} \boldsymbol{B} \quad \boldsymbol{\Omega}=\boldsymbol{M C} \\
\\
\boldsymbol{\Lambda}=\boldsymbol{M}^{-1} \boldsymbol{A} \boldsymbol{M}=\operatorname{diag}\left(\lambda_{i}\right)
\end{aligned}
$$

la matrice $\boldsymbol{M}$ étant la matrice des $n$ vecteurs propres linéairement indépendants (matrice modale).

Les avantages suivants apparaissent alors :

la représentation (3) est particulièrement bien adaptée aux résolutions analytique et numérique du fait de l'indépendance de chaque composante du vecteur d'état,

le critère de stabilité asymptotique du système est aisément vérifiable : les valeurs propres doivent être toutes à partie réelle négative,

en cas de discrétisation temporelle, l'examen des constantes de temps du système $\tau_{i}=-1 / \operatorname{Re}\left(\lambda_{i}\right)$ permet un ajustement du pas de temps.

Pour les systèmes d'ordre élevé ( $n$ grand), les formulations (1) (2) et (3) deviennent particulièrement lourdes si on veut faire de nombreuses simulations ou si on désire les incorporer dans des algorithmes de contrôle-commande de processus. Dans de tels cas, l'intérêt de disposer d'un modèle de faible dimension est certain et les formulations (2) et (3), qui présentent l'avantage de mettre en évidence les paramètres d'entrée et de sortie du système, sont alors plus spécialement adaptées aux techniques de réduction. L'objectif de ces méthodes est d'obtenir un modèle de faible dimension $(m \ll n)$ avec une structure semblable à celle du modèle d'origine.

Dans cet article, relatif à la réduction de modèles décrits par l'équation (1), nous proposons :

i) d'exposer différentes formulations des équations d'état en commentant leur adéquation au problème de la réduction,

ii) de présenter une méthode originale d'obtention d'un modèle modal de faible dimension par identification de ses modes dominants,

iii) de comparer différentes techniques de réduction à cette méthode sur un exemple de modélisation d'un plancher chauffant.

\section{Représentation d'état et réduction.}

Nous présentons dans ce paragraphe différentes formulations des équations d'état (2), en introduisant 
les dérivées successives du vecteur d'entrée $u(t)$ et nous discutons de l'intérêt de ces représentations pour la réduction de modèle.

Sous l'hypothèse $u$ dérivable par rapport au temps (de classe $C^{1}$ ), effectuons le changement de variables $x(t)=x_{1}(t)-\boldsymbol{A}^{-1} \boldsymbol{B} u(t)$ dans les équations (2). Il résulte alors la reformulation suivante des équations d'état :

$$
\begin{aligned}
& \dot{x}_{1}=\boldsymbol{A} x_{1}+\boldsymbol{B}_{1} \dot{u} \\
& y=\boldsymbol{C} x_{1}+\boldsymbol{D}_{1} u
\end{aligned}
$$

avec: $\boldsymbol{B}_{1}=\boldsymbol{A}^{-1} \boldsymbol{B}$ et $\boldsymbol{D}_{1}=-\boldsymbol{C A}^{-1} \boldsymbol{B}$.

Un raisonnement analogue peut être appliqué à la formulation (4) avec le changement de variables : $x_{1}(t)=x_{2}(t)-\boldsymbol{A}^{-1} \boldsymbol{B}_{1} u(t)$. En supposant maintenant $u$ dérivable $i$ fois (de classe $C^{i}$ ), de façon récurrente, après $i$ changements de variables semblables, on a :

$$
\begin{aligned}
\dot{x}_{i} & =\boldsymbol{A} x_{i}+\boldsymbol{B}_{i} u^{(i)} \\
y & =\boldsymbol{C} x_{i}+\Sigma \boldsymbol{D}_{j} u^{(j-1)} \quad(1 \leqslant j \leqslant i)
\end{aligned}
$$

avec : $\boldsymbol{B}_{i}=\boldsymbol{A}^{-i} \boldsymbol{B} \quad \boldsymbol{D}_{j}=-\boldsymbol{C A}^{-j} \boldsymbol{B}$

$\boldsymbol{u}^{(i)}$ étant la dérivée d'ordre $i$ de $u$ par rapport au temps. Cette formulation fait ressortir les paramètres de Markov $\boldsymbol{D}_{j}$ intervenant dans le développement en série de la matrice de transfert, dans le cas d'une représentation de type externe : avec $s$ la variable de Laplace, à partir des relations (2) la matrice de transfert $\mathbf{H}(s)$ s'écrit :

$$
\begin{aligned}
& \mathbf{H}(s)=\boldsymbol{C}(s \mathbf{1}-\boldsymbol{A})^{-1} \boldsymbol{B}=\Sigma-\boldsymbol{C A}^{-j} \boldsymbol{B} s^{j} \\
&(1 \leqslant j<\infty) .
\end{aligned}
$$

Cette description est exploitée tant en identification [20] qu'en réduction de modèle [1, 21], le principe étant de trouver une réalisation minimale dans l'espace de Laplace.

En ce qui concerne la représentation interne, les techniques de réduction de modèles sont appliquées à la forme (2) $[1,11,12]$. Elles visent alors à l'obtention d'un modèle d'état de faible dimension s'écrivant sous la forme :

$$
\begin{aligned}
& \dot{\mathbf{x}}(t)=\mathbf{A x}(t)+\mathbf{B} u(t) \\
& \hat{y}(t)=\mathbf{C x}(t)
\end{aligned}
$$

où $\mathrm{x}(\operatorname{dim} . m \ll n)$ est le nouveau vecteur d'état, $\hat{y}$ l'estimation des sorties, A, B et $\mathbf{C}$ les matrices réduites correspondantes.

Ces mêmes techniques peuvent alors être appliquées à la formulation (5) et permettront d'obtenir des matrices $\mathbf{A}, \mathbf{B}_{\mathbf{i}}, \mathbf{C}$ ainsi qu'une estimation $\hat{y} \mathrm{du}$ vecteur des sorties selon:

$$
\begin{aligned}
& \dot{\mathbf{x}}_{i}=\mathbf{A} \mathbf{x}_{i}+\mathbf{B}_{\mathrm{i}} u^{(i)} \\
& \hat{y}=\mathbf{C x}_{i}+\Sigma \boldsymbol{D}_{j} u^{(j-1)} \quad(1 \leqslant j \leqslant i) .
\end{aligned}
$$

L'avantage théorique de la formulation (7) est la conservation de régimes forcés de plus en plus complexes ; ainsi, une méthode de réduction appliquée à :

- la formulation (4) conserve naturellement les régimes stationnaires $u=$ constante ;

- la formulation (5) conserve toute entrée telle que $u^{(k)}=0$ pour $k>\mathrm{i}$.

Le principal inconvénient est l'adjonction de termes supplémentaires qui alourdissent le calcul au niveau de la sortie du modèle réduit.

Remarque 1 : Quand on choisit la réduction élémentaire $x_{i}=0$, on retrouve la formulation du modèle réduit proposé en [13]:

$$
\hat{y}=\Sigma \boldsymbol{D}_{j} u^{(j-1)} \quad(1 \leqslant j \leqslant i) .
$$

Remarque 2: Avec $i=1$, c'est-à-dire si la représentation (4) est choisie, on obtient l'équation d'état utilisée en [9] qui s'appuie sur la notion de régime glissant :

$$
x_{\mathrm{gl}}(t)=-\boldsymbol{A}^{-1} \boldsymbol{B} u(t) .
$$

Ce régime glissant correspond à l'état qu'aurait le système en l'absence d'inertie thermique.

La représentation (4) offre par ailleurs deux avantages particulièrement intéressants :

a) en général, les méthodes de réduction appliquées à la formulation (2) nécessitent l'introduction d'une matrice de couplage entrées-sorties $\mathbf{D}$ dans le modèle réduit afin de préserver les états stationnaires, l'équation (6.2) s'écrivant alors :

$$
\hat{y}(t)=\mathbf{C x}(t)+\mathbf{D} u(t) .
$$

Cette propriété indispensable pour les problèmes de réduction, est naturellement obtenue quand la réduction porte sur la formulation (4).

b) Elle peut être étendue à des problèmes non linéaires visant la prise en compte de l'évolution de paramètres thermophysiques avec la température. En effet, si un modèle statique $y_{\mathrm{s}}=f(u)$ de ce système non linéaire est utilisé, son comportement dynamique peut être approché par une représentation telle que :

$$
\begin{aligned}
& \dot{x}=\boldsymbol{A} x+\boldsymbol{B}_{1} \dot{u} \\
& y=\boldsymbol{C} x+f(u)
\end{aligned}
$$

conservant tout régime stationnaire.

Ces avantages nous ont fait retenir la représentation d'état sous la formulation (4). Nous nous intéressons ci-dessous à l'obtention d'une telle représentation, quand elle est exprimée dans la base modale. Notons en effet que les développements présentés dans ce paragraphe auraient pu être effectués sur la forme canonique (3). 
3. Modes dominants d'un système : sélection et identification.

De nombreuses méthodes de réduction utilisent les " modes dominants » sélectionnés dans le spectre de la matrice d'évolution $\boldsymbol{A}$. Il est alors nécessaire de définir une méthodologie de sélection de ces modes et de nombreux auteurs s'y sont employés. De manière non exhaustive, nous allons succinctement évoquer quelques approches, puis nous présenterons une méthode d'obtention de modes dominants par identification, méthode dont l'avantage est précisément d'éviter le difficile problème de la sélection.

\subsection{RÉDUCTION MODALE PAR SÉLECTION DE MODES} DOMINANTS. ANALYSE SPECTRALE. - En reprenant les équations d'état dans la base modale (3), le problème de la réduction est de trouver une représentation d'état modale du type :

$$
\begin{aligned}
& \dot{\xi}=\boldsymbol{\Lambda} \xi+\boldsymbol{\Gamma} u \\
& \hat{y}=\mathbf{\Omega} \xi+\mathbf{D} u
\end{aligned}
$$

où se trouvent groupées dans $\boldsymbol{\Lambda}$ les $m$ valeurs propres sélectionnées; $\xi$ est le vecteur d'état réduit de dimension $\mathrm{m}(m \ll n)$ correspondant en fait à une partition du vecteur d'état d'origine $\xi$, la matrice $\boldsymbol{\Gamma}(\operatorname{dim} . m, p)$ est issue du même partitionnement relatif à $\boldsymbol{\Gamma}, \boldsymbol{\Omega}(\operatorname{dim} . q, m)$ est la matrice d'observation réduite et $\mathbf{D}$ est une matrice de couplage entrées-sorties assurant la conservation des régimes stationnaires (cf. (8)).

Le problème est donc de sélectionner les $m$ valeurs propres et de déterminer les matrices d'observation et de couplage correspondantes. Parmi les méthodes modales, citons les plus classiques:

a) une catégorie de méthodes utilise la conservation de $m$ valeurs propres correspondant aux $\mathrm{m}$ constantes de temps les plus grandes; les $(n-m)$ composantes restantes du vecteur d'état sont supposées atteindre leur régime asymptotique de façon instantanée. Ce type de réduction a notamment été utilisé dans les références $[2,3,10]$;

b) pour la méthode d'agrégation la sélection des modes se fait en fonction de leur contribution énergétique lorsque le système est soumis à des entrées tests (type échelon par exemple) sur chacune des composantes du vecteur des entrées. Ces travaux ont en particulier été développés dans les références $[4,14]$;

c) si on utilise les notions de commandabilité (via la matrice $\boldsymbol{\Gamma}$ ) et d'observabilité (via la matrice $\boldsymbol{\Omega})$, l'analyse systématique de l'influence de chaque entrée sur chaque sortie est également possible. En excitant successivement chaque composante du vecteur d'entrée (classiquement un échelon), la contribution de chaque valeur propre au niveau de la sortie permet de déterminer les plus influentes qui sont alors retenues. Ces méthodes ont particulièrement été utilisées dans les références $[5,8,9,15]$.

3.2 Modèle MODAL IDENTIFIÉ. - La diversité des approches évoquées ci-dessus atteste de la difficulté de sélectionner $\mathrm{m}$ modes propres dans le spectre de la matrice d'évolution. De plus, la possibilité d'un amalgame de diverses valeurs propres est également envisageable [4, 14], mais pose un problème encore plus délicat. C'est la raison pour laquelle, suite à des travaux déjà entrepris sur l'identification d'un modèle modal [16], nous proposons ci-après une méthode de réduction mettant en évidence les modes prépondérants par identification et non pas en suivant une méthodologie de sélection dans le spectre.

Etant donné les avantages précédemment montrés de la formulation (9), nous adoptons cette structure pour le modèle réduit; dans la base modale on a :

$$
\begin{aligned}
& \dot{\xi}=\mathbf{\Lambda} \xi+\Gamma \dot{u} \\
& \dot{y}=\mathbf{\Omega} \xi+f(u)
\end{aligned}
$$

où $\xi$ est le vecteur d'état $(\operatorname{dim} . m) \boldsymbol{\Lambda} \boldsymbol{\Gamma}$ et $\boldsymbol{\Omega}$ sont respectivement la matrice diagonale des $m$ valeurs propres, les matrices de commande et d'observation à identifier, $\hat{y}$ est l'estimation du vecteur des sorties, $f(u)$ est la sortie correspondant au régime glissant.

3.2.1 Identification des modes dominants. - Dans un premier temps, les matrices $\boldsymbol{\Lambda}$ et $\boldsymbol{\Omega}$ sont obtenues par identification lors d'une comparaison entre les sorties du modèle complet $y(t)$ et du modèle réduit $\hat{y}(t)$, lorsque le système évolue d'un état initial $\xi(0)$ à un état asymptotique correspondant à $u=$ constante.

Pour $u=$ constante, $\dot{u}=0$ et (11) s'intègre sous la forme :

$$
\hat{y}-f(u)=\mathbf{\Omega} \exp (\mathbf{\Lambda} t) \xi(0)
$$

Rangeons les $m$ composantes du vecteur $\xi(0)$ sur la diagonale d'une matrice carrée $(m, m)$ telle que $\boldsymbol{\xi}_{0}=\operatorname{diag}\left[\xi_{i}(0)\right]$ et avec 1 le vecteur de dimension $m$ et de composantes unitaires (12) s'écrit :

$$
\hat{y}-f(u)=\mathbf{\Omega} \exp (\boldsymbol{\Lambda} t) \boldsymbol{\xi}_{0} \underline{1} .
$$

Les matrices $\exp (\boldsymbol{\Lambda} t)$ et $\boldsymbol{\xi}_{0}$ sont diagonales et leur produit est donc commutatif. L'équation (13) peut s'écrire alors sous la forme :

$$
\hat{y}-f(u)=\mathbf{K} Z^{\mathrm{t}}
$$

avec $\mathbf{K}$ la matrice telle que $\mathbf{K}=\boldsymbol{\Omega} \boldsymbol{\xi}_{0}$ et la notation $Z^{\mathrm{t}}$ pour le vecteur de dimension $m$ tel que sa $i$-ième composante soit $\left(Z^{\mathrm{t}}\right)_{i}=Z_{i}^{\mathrm{t}}$ où $Z_{i}=\exp \left(\lambda_{i}\right)$. La minimisation d'une norme de l'écart entre les sorties 
du modèle complet et de l'équation (14), permet alors :

- l'estimation de la dimension du modèle réduit,

- l'identification des valeurs propres,

- l'identification de la matrice $\mathbf{K}$.

Le traitement numérique de ce problème d'optimisation est décrit en annexe 1 .

Remarque : en pratique, dans cette phase de la procédure, pour obtenir une meilleure identification, on travaille avec un vecteur de sortie étendu permettant l'observation des variations d'un grand nombre, voire de toutes les températures $(q=n)$.

3.2.2 Détermination de la matrice de sortie. - Pour obtenir la matrice de sortie $\boldsymbol{\Omega}$, d'après les résultats obtenus ci-dessus, on remarque qu'avec le changement de variables $\xi=\xi_{0} \xi^{\prime}$, l'équation (11) devient :

$$
\begin{aligned}
& \dot{\xi}^{\prime}=\Lambda \xi^{\prime}+\Gamma^{\prime} \dot{u} \quad \text { avec } \quad \boldsymbol{\Gamma}^{\prime}=\xi_{0}^{-1} \boldsymbol{\Gamma} \\
& \hat{y}=\mathbf{K} \xi^{\prime}+f(u)
\end{aligned}
$$

Cette représentation d'état étant semblable à la représentation (11), on en déduit le résultat essentiel suivant : la matrice $\mathbf{K}$ précédemment identifiée peut être utilisée comme matrice d'observation $\boldsymbol{\Omega}$. En gardant les notations $\xi$ pour $\xi^{\prime}$ et $\boldsymbol{\Gamma}$ pour $\boldsymbol{\Gamma}^{\prime}$, on peut ainsi conserver la formulation (11).

3.2.3 Détermination de la matrice d'entrée. - Pour déterminer la matrice d'entrée $\boldsymbol{\Gamma}$, il est naturel de chercher à minimiser l'écart entre les sorties des modèles complet et identifié sur des entrées spécifiques $u(t)$. Considérant une norme de l'écart entre $\hat{y}(t)$ et $y(t)$, le problème se pose sous la forme de la minimisation d'un critère du type :

$$
J=\int_{0}^{\infty}\|\hat{y}(t)-y(t)\|^{2} \mathrm{~d} t .
$$

Classiquement, des considérations statistiques conduisent à retenir une norme quadratique [17] :

$$
\|\hat{y}(t)-y(t)\|^{2}=[\hat{y}(t)-y(t)]^{\mathrm{T}} \mathbf{P}(t)[\hat{y}(t)-y(t)]
$$

où $\mathbf{P}(t)$ est une matrice de pondération $(\operatorname{dim} . q, q)$ définie positive pour tout $t$.

Comme entrées spécifiques, nous avons choisi des échelons unitaires appliqués indépendamment sur chaque composante du vecteur $u$. Les solutions analytiques de (9) et (11) permettent alors d'exprimer les écarts entre les sorties sous la forme :

$$
\boldsymbol{\varepsilon}(t)=\left[\boldsymbol{\Omega} \exp (\boldsymbol{\Lambda} t) \boldsymbol{\Gamma}-\boldsymbol{C} \exp (\boldsymbol{A} t) \boldsymbol{A}^{-1} \boldsymbol{B}\right](17)
$$

où $\varepsilon$ est la matrice des écarts entre $\hat{y}(t)$ et $y(t)$ telle que $\varepsilon_{i j}=\hat{y}_{i}(t)-y_{i}(t)$ quand on considère un échelon sur la composante $u_{j}$. Comme prévu, si $t$

\footnotetext{
REVUE DE PHYSIQUE APPLIQUÉE. - T. 25, N 8, AOÛT 1990
}

est infini, alors $\boldsymbol{\varepsilon}(t)=\mathbf{0}$ (conservation des régimes asymptotiques).

Dans le cadre de ce travail, pour la matrice de pondération, nous avons choisi $\mathbf{P}(t)=\delta(t) 1$, où $\delta(t)$ est la distribution de Dirac et 1 la matrice identité (dim. $q, q$ ) ; ce choix signifie que l'on exige un écart minimum entre les modèles complet et identifié à l'instant de l'échelon. La minimisation du critère $\mathbf{J}$ défini par (15) se traduit alors simplement par la résolution de :

$$
\mathbf{\Omega} \boldsymbol{\Gamma}=\boldsymbol{C A}^{-1} \boldsymbol{B} .
$$

La matrice inconnue $\Gamma$ est en fait constituée de $m p$ éléments alors que la matrice $\boldsymbol{C A}^{-1} \boldsymbol{B}$ en possède $q p$. Si $q<m$, l'équation (18) est inexploitable car le système est sous-déterminé et si $q=m$, elle constitue un système de $q p$ équations à $q p$ inconnues. Dans le cas plus fréquent où $q>m$, le système est surdéterminé et la résolution de (18) peut s'obtenir au sens des moindres carrés. On trouve alors :

$$
\boldsymbol{\Gamma}=\left(\boldsymbol{\Omega}^{\mathrm{T}} \boldsymbol{\Omega}\right)^{-1} \boldsymbol{\Omega}^{\mathrm{T}} \boldsymbol{C} \boldsymbol{A}^{-1} \boldsymbol{B} .
$$

Dans le paragraphe suivant, nous présentons l'application de cette méthode en traitant d'un cas concret : la modélisation d'un plancher chauffant et nous comparons nos résultats à ceux provenant d'autres techniques de réduction.

\section{Le système thermique étudié : un plancher chauf- fant.}

DESCRIPTION ET MODÉLISATION. - Le dispositif étudié est un plancher chauffant sur vide sanitaire, dont on a représenté une coupe en figure 1 . Ce système multicouche est constitué :

- d'un carrelage d'épaisseur $1 \mathrm{~cm}$

$\left(k=1,04 \mathrm{~W} \mathrm{~m}^{-1} \mathrm{~K}^{-1}, \rho c=1,05 \times 10^{+6} \mathrm{~J} \mathrm{~m}^{-3} \mathrm{~K}^{-1}\right)$,

- d'une chappe en ciment d'épaisseur $7 \mathrm{~cm}$

$\left(k=1,15 \mathrm{~W} \mathrm{~m}^{-1} \mathrm{~K}^{-1}, \rho c=1,64 \times 10^{+6} \mathrm{~J} \mathrm{~m}^{-3} \mathrm{~K}^{-1}\right)$

dans laquelle se trouve noyé un tube $(\phi=16 \mathrm{~mm})$ dans lequel circule le fluide calorporteur,

- d'une couche d'isolant d'épaisseur $4 \mathrm{~cm}$ $\left(k=0,04 \mathrm{~W} \mathrm{~m}^{-1} \mathrm{~K}^{-1}, \rho c=5 \times 10^{+4} \mathrm{~J} \mathrm{~m}^{-3} \mathrm{~K}^{-1}\right)$,

- d'une dalle porteuse en béton d'épaisseur $10 \mathrm{~cm}$

$k=1,5 \mathrm{~W} \mathrm{~m}{ }^{-1} \mathrm{~K}^{-1}, \rho c=2,21 \times 10^{+6} \mathrm{~J} \mathrm{~m}^{-3} \mathrm{~K}^{-1}$ ).

Les hypothèses retenues et les conditions aux limites du problème sont les suivantes :

- les flux sont nuls sur les axes de symétrie (conditions de Neumann homogènes),

- aux interfaces carrelage-air, ciment-fluide, et béton-air, les transferts thermiques sont décrits par des coefficients de transfert constants (conditions de Fourier), respectivement : $h_{i}=5,9 \mathrm{~W} \mathrm{~m}^{-2} \mathrm{~K}^{-1}$, 


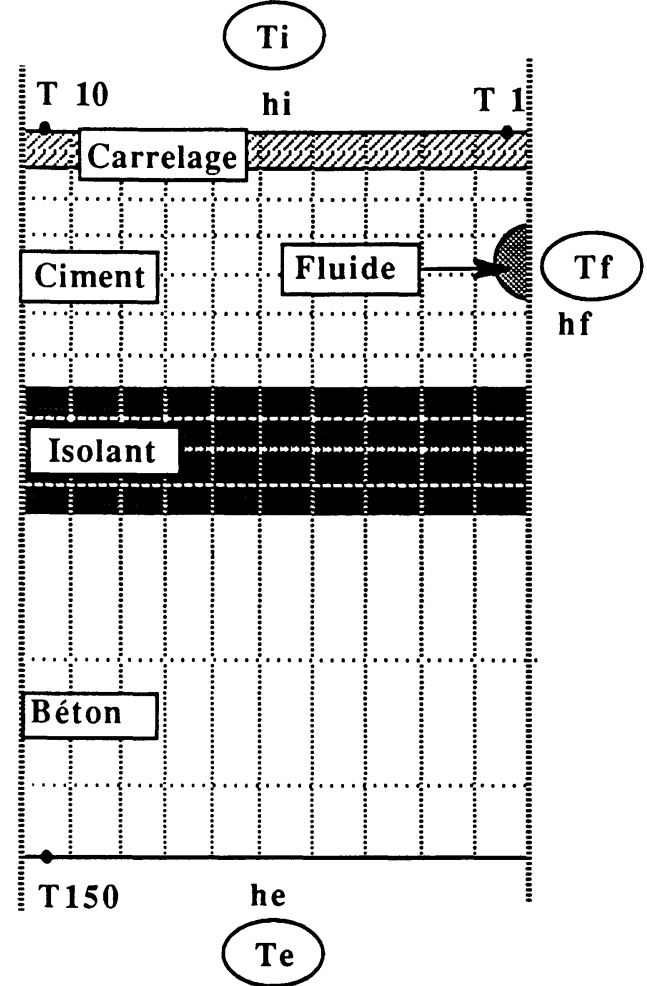

Fig. 1. - Schéma du plancher: maillage, conditions limites et les 3 températures $T_{1}, T_{10}$ et $T_{150}$.

[Floor draft : the mesh, the boundary conditions and the 3 temperatures $T_{1}, T_{10}$ and $T_{150}$.]

$h_{\mathrm{f}}=5000 \mathrm{~W} \mathrm{~m}^{-2} \mathrm{~K}^{-1}, h_{\mathrm{e}}=5,9 \mathrm{~W} \mathrm{~m}^{-2} \mathrm{~K}^{-1}$, relativement aux températures d'air intérieur $T_{\mathrm{i}}$, de fluide $T_{\mathrm{f}}$ et d'air extérieur $T_{\mathrm{e}}$.

Afin de mettre le modèle sous la forme (1), nous avons utilisé la méthode nodale. Un maillage permettant de faire varier le nombre de nœuds horizontalement et verticalement dans chaque couche a été mis en place et a permis, grâce à diverses simulations en régime transitoire, de fixer un maillage convenable $(10 \times 15=150$ nœuds $)$.

Sous la forme (2), ce sont les 3 températures $T_{\mathrm{i}}, T_{\mathrm{f}}, T_{\mathrm{e}}$ qui constituent les composantes du vecteur d'entrée $u$, et comme composantes du vecteur des sorties, nous avons retenu les températures des 2 nœuds extrêmes à la surface supérieure $\left(T_{1}\right.$ et $T_{10}$ ) qui matérialisent le gradient thermique longitudinal et une température à la surface inférieure $\left(T_{150}\right)$ où la température est quasiuniforme étant donné la présence de l'isolant. Les matrices de la représentation (2) sont alors bien définies.

\section{Expérimentations numériques et résultats.}

5.1 Le Modèle Complet (MC). - Le modèle complet à 150 nœuds est le modèle qui nous sert de référence pour les différentes simulations. Une analyse du spectre de la matrice d'évolution $\boldsymbol{A}$ montre que les 150 valeurs propres correspondent à des constantes de temps s'échelonnant entre $35784 \mathrm{~s}$ et $9,5 \mathrm{~s}$ : les 5 premières sont (en s): 35784,8837 , $3472,1655,1559$, les 100 dernières sont comprises entre 100 et $9 \mathrm{~s}$. Conformément à ce qui a été dit en introduction, l'examen de ces constantes de temps incite à choisir un pas de temps de l'ordre de quelques secondes ( $3 \mathrm{~s}$ en pratique). Pour l'intégration sur le temps, un schéma aux différences finies du second ordre (Crank-Nicholson) a été adopté.

5.2 Le Modèle Identifié (MI). - Pour identifier les matrices $\boldsymbol{\Lambda}$ et $\boldsymbol{\Omega}$ du modèle réduit (cf. 3.2.1), une simulation de type relaxation a été faite sur le Modèle Complet (MC). Un champ thermique spatial initial a été obtenu par une simulation avec un vecteur d'entrée de composantes $(10,40,0)$, en ${ }^{\circ} \mathrm{C}$ le champ asymptotique final correspond au vecteur d'entrée $(10,5,0)$. Les 150 thermogrammes temporels de relaxation, comprenant chacun 200 températures, ont été utilisés pour identifier les modes dominants et la matrice d'observation $\boldsymbol{\Omega}$. Pour quantifier la qualité de l'identification en fonction du nombre de modes dominants identifiés $m$, nous présentons dans le tableau I, l'écart quadratique moyen $\sigma$ (en ${ }^{\circ} \mathrm{C}$ entre les 150 thermogrammes issus de MC et de MI pour différentes valeurs de $m$.

Tableau I.

\begin{tabular}{|c|c|c|c|c|c|}
\hline$m$ & 1 & 2 & 3 & 4 & 5 \\
\hline$\sigma$ & 0.721 & 0.373 & 0.186 & 0.037 & 0.004 \\
\hline
\end{tabular}

Sur ce tableau apparaît très clairement la décroissance de $\sigma\left(\sigma^{2}=C(m) /(r q)\right.$ cf. Annexe 1) en fonction de l'accroissement de l'ordre du modèle identifié.

A titre d'exemple, on a représenté sur les figures 2 à 5 les thermogrammes correspondant aux nœuds 1 , 10 et 150 respectivement ajustés avec $1,2,3$ et 4 exponentielles exprimées sous forme de constantes de temps. L'incrémentation à $m=5$ ne fournissant pas une amélioration sensible au niveau des thermogrammes correspondants, le modèle réduit adopté est arrêté à la dimension 4.

La matrice d'entrée a été également déterminée avec les 150 thermogrammes à partir de la relation (19). Finalement, le modèle réduit identifié est donc d'ordre 4 et se présente sous la forme (en ne faisant figurer que les 3 températures $T_{1} T_{10} T_{150}$ dans le vecteur de sorties): 


$$
\begin{gathered}
\dot{\xi}(t)=\left|\begin{array}{cccc}
-1 / \tau_{1} & 0 & 0 & 0 \\
0 & -1 / \tau_{2} & 0 & 0 \\
0 & 0 & -1 / \tau_{3} & 0 \\
0 & 0 & 0 & -1 / \tau_{4}
\end{array}\right| \xi(t)+\left|\begin{array}{rrr}
-3,27 \times 10^{-3} & -2,86 \times 10^{-2} & -1,95 \times 10^{-4} \\
5,00 \times 10^{-4} & -2,86 \times 10^{-2} & 4,09 \times 10^{-4} \\
-2,02 \times 10^{-2} & -2,86 \times 10^{-2} & 1,54 \times 10^{-3} \\
-1,18 \times 10^{-2} & -2,86 \times 10^{-2} & -1,79 \times 10^{-1}
\end{array}\right| \dot{u}(t) \\
\hat{y}(t)=\left|\begin{array}{rrrr}
16,32 & 0,69 & 6,38 & 0,25 \\
-8,48 & 2,05 & 0,44 & 0,57 \\
-0,12 & 0,08 & -1,15 & 4,20
\end{array}\right| \xi(t)+\left|\begin{array}{rrrr}
0,3077 & 0,6731 & 0,0191 \\
0,5295 & 0,4154 & 0,0551 \\
0,0414 & 0,0860 & 0,8726
\end{array}\right| u(t)
\end{gathered}
$$

avec :

$$
\tau_{1}=35332 \mathrm{~s}, \quad \tau_{2}=8840 \mathrm{~s}, \quad \tau_{3}=1511 \mathrm{~s}, \quad \tau_{4}=297 \mathrm{~s}
$$

Identification 1 exponentielle

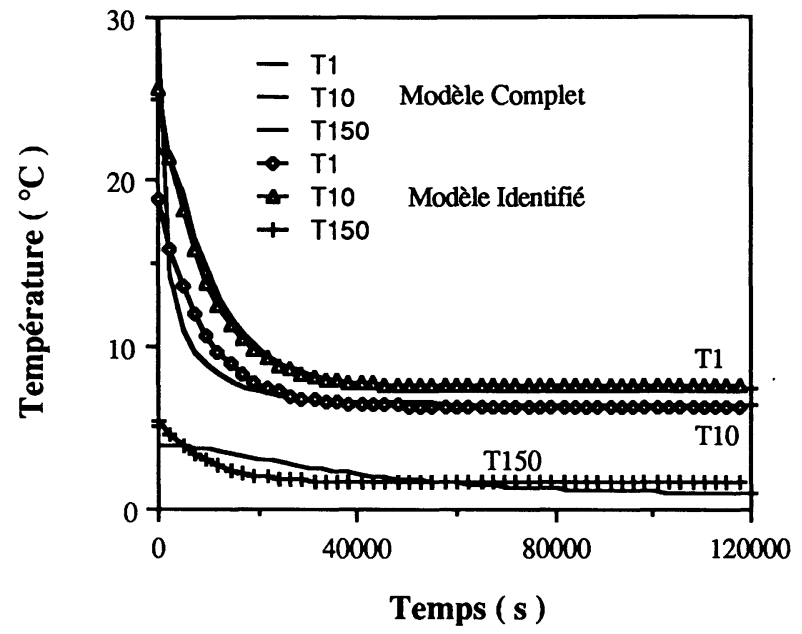

Fig. 2. - Relaxation : modèle complet et modèle identifié avec une constante de temps : $\tau_{1}=9118 \mathrm{~s}$.

[Relaxation: complete model and identified one whith one time constant : $\tau_{1}=9118 \mathrm{~s}$.]

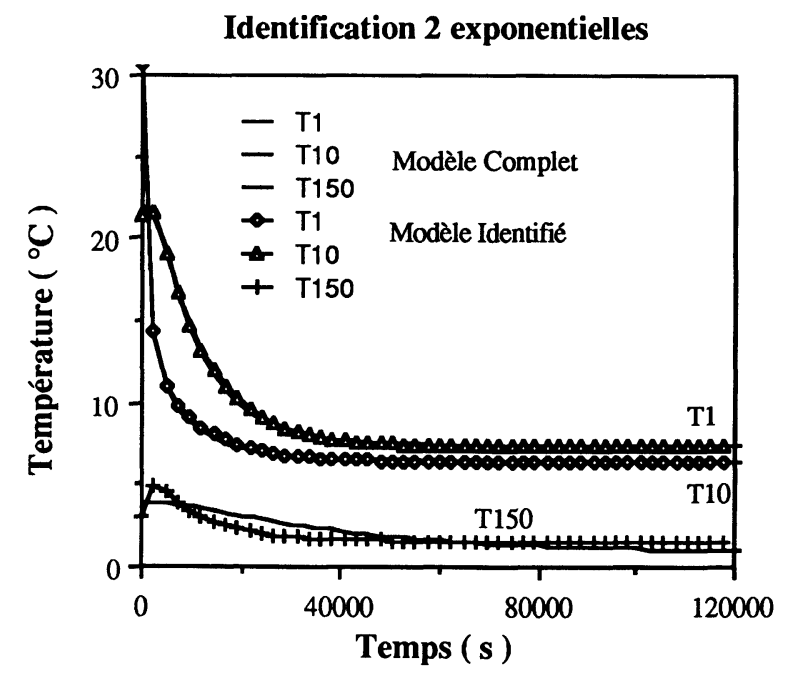

Fig. 3. - Relaxation : modèle complet et modèle identifié avec deux constantes de temps: $\tau_{1}=10104 \mathrm{~s}$, $\tau_{2}=1225 \mathrm{~s}$.

[Relaxation: complete model and identified one whith two time constants : $\tau_{1}=10104 \mathrm{~s}, \tau_{2}=1225 \mathrm{~s}$.]

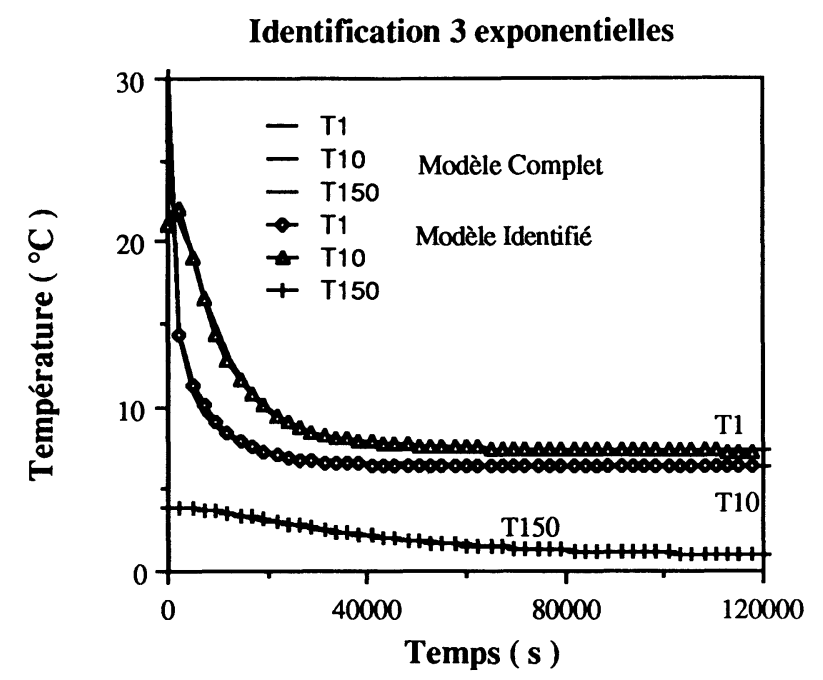

Fig. 4. - Relaxation : modèle complet et modèle identifié avec trois constantes de temps: $\tau_{1}=31852 \mathrm{~s}$, $\tau_{2}=8556 \mathrm{~s}, \tau_{3}=989 \mathrm{~s}$.

[Relaxation: complete model and identified one whith three time constants: $\tau_{1}=31852 \mathrm{~s}, \quad \tau_{2}=8556 \mathrm{~s}$, $\tau_{3}=989 \mathrm{~s}$.]

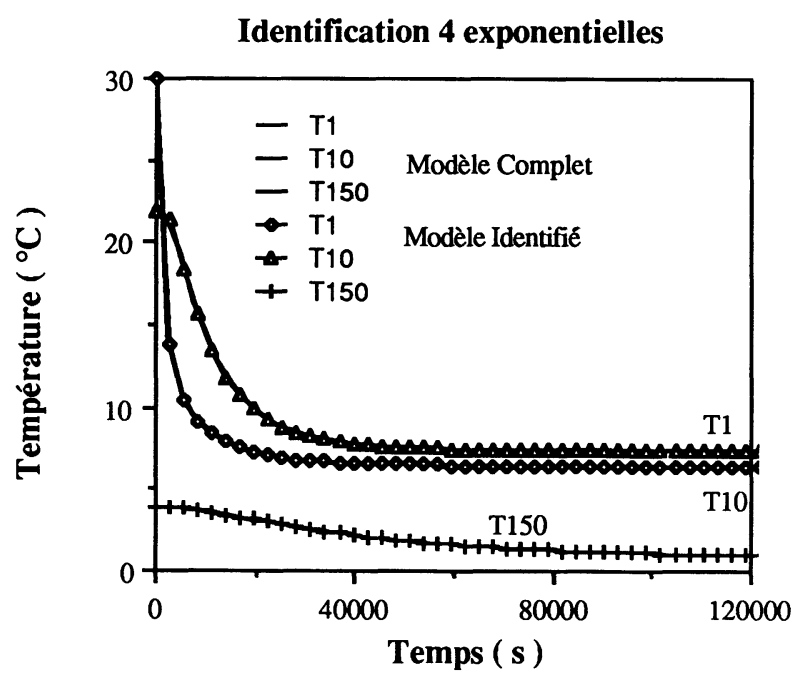

Fig. 5. - Relaxation : modèle complet et modèle identifié avec quatre constantes de temps: $\tau_{1}=35332 \mathrm{~s}$, $\tau_{2}=8540 \mathrm{~s}, \tau_{3}=1511 \mathrm{~s}, \tau_{4}=297 \mathrm{~s}$.

[Relaxation: complete model and identified one whith four time constants: $\tau_{1}=35332 \mathrm{~s}, \quad \tau_{2}=8540 \mathrm{~s}$, $\tau_{3}=1511 \mathrm{~s}, \tau_{4}=297 \mathrm{~s}$.] 
Si on compare ces 4 constantes de temps à celles issues du spectre de la matrice $\mathbf{A}$, on constate que $\tau_{1}$ et $\tau_{2}$ correspondent aux 2 premières des $150 \mathrm{du}$ MC (respectivement de 35784 et 8837 s) : il apparait donc ici que l'identification "sélectionne" des modes prépondérants préexistants. Les 2 autres valeurs $\tau_{3}$ et $\tau_{4}$ correspondent alors à un "amalgame » des 148 autres constantes et c'est précisément dans la possibilité de cet "amalgame " que se situe l'intérêt essentiel de la méthode proposée.

5.3 Simulations ET COMPARAISON AVEC D'AUTRES MÉTHODES. - Pour tester le modèle identifié et le comparer à d'autres modèles réduits, nous utilisons ici 3 autres méthodes de réduction que nous avons déjà mises en œuvre lors de travaux précédents [12]: 2 autres méthodes modales Marshall [2] (dominances de temps caractéristiques) et agrégation [4] (critères énergétiques associés à chaque mode) - évoquées au paragraphe 3.1 ainsi que la technique d'Eitelberg [11] dont le principe est de sélectionner $m$ composantes du vecteur d'état et d'obtenir un modèle réduit de la forme (6) en minimisant la norme d'une erreur d'équation. Soulignons ici que ces méthodes ont été utilisées sous leur forme première, c'est-à-dire sans chercher à améliorer les résultats par un quelconque affinement (cf. par exemple [18] pour la méthode Eitelberg).

5.3.1 Simulation de type échelon. - Une première simulation est faite avec un échelon sur la température du fluide caloporteur $T_{\mathrm{f}}$ qui passe de 5 à $40{ }^{\circ} \mathrm{C}$ tandis que les températures $T_{\mathrm{i}}$ et $T_{\mathrm{e}}$ restent inchangées, respectivement 10 et $0{ }^{\circ} \mathrm{C}$. Pour toutes les méthodes, la dimension du modèle réduit a été fixée à 4. Pour les 3 nœuds 1,10 et 150 , les résultats Eitelberg et Marshall sont présentés en figure 6,

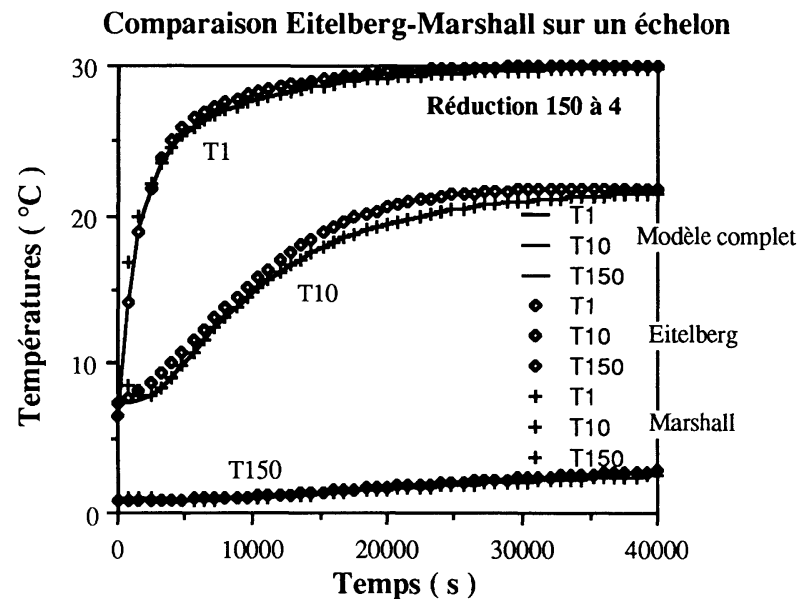

Fig. 6. - Comparaison du modèle complet et des modèles de Marshall et d'Eitelberg sur un échelon.

[Comparison between the complete model and the reduced models of Marshall and Eitelberg according to a step.] l'agrégation et MI sont présentés en figure 7. On constate sur ces figures la bonne concordance entre tous les modèles réduits et le modèle complet. On peut cependant noter que :

- la 'méthode d'Eitelberg donne ici un résultat globalement moins satisfaisant, bien que peu perturbé au voisinage de $t=0$ par rapport aux autres méthodes,

- les méthodes de Marshall et d'agrégation approchent très bien le modèle complet pour $t>5000 \mathrm{~s}$, mais de légères perturbations apparaissent au voisinage de $t=0$ (notamment T10 pour Marshall, T10 et T150 pour l'agrégation),

- le modèle identifié (MI) donne des résultats très satisfaisants, malgré une légère perturbation à $t=0$ pour $\mathrm{T} 10$.

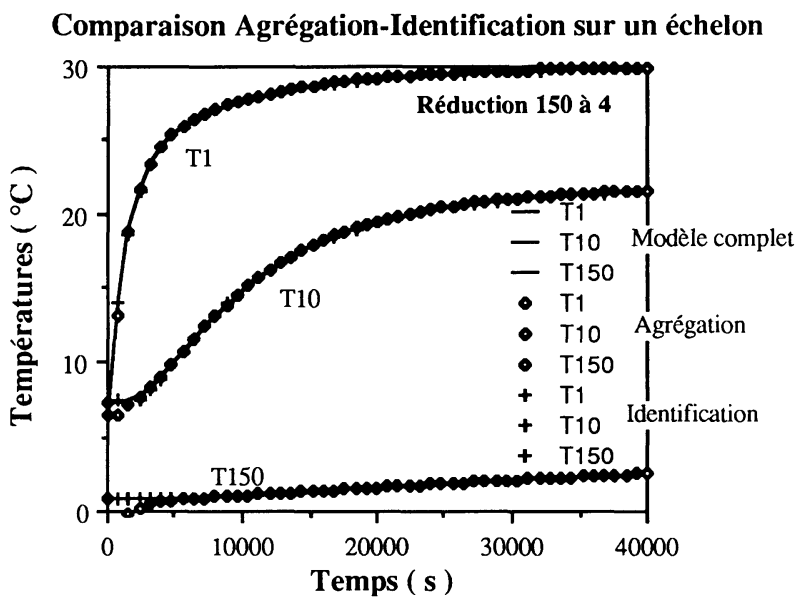

Fig. 7. - Comparaison du modèle complet et des modèles agrégé et identié sur un échelon.

[Comparison of the complete model and the agregated and identified ones accordint to a step.]

5.3.2 Simulation "Jour-Nuit». - Pour tester le modèle identifié et les autres modèles réduits dans une situation un peu plus complexe, nous avons fait varier toutes les composantes du vecteur d'entrée: nous avons simulé un refroidissement brutal des températures intérieure et extérieure - simulant un passage jour-nuit - puis une augmentation de la température du fluide selon une rampe. Ceci est résumé dans le tableau II où figure le vecteur des entrées en fonction du temps :

Tableau II.

\begin{tabular}{|c|c|c|c|c|}
\hline$t(\times 1000 \mathrm{~s})$ & $<0$ & $\begin{array}{c}0 \\
\text { à } 20\end{array}$ & 20 à 22 & 22 à 40 \\
\hline$T_{\mathrm{i}}$ & 20 & 10 & 10 & 10 \\
$T_{\mathrm{f}}$ & 30 & 30 & $30+5(\mathrm{t}-20)$ & 40 \\
$T_{\mathrm{e}}$ & 15 & 5 & 5 & 5 \\
\hline
\end{tabular}


Les résultats correspondant aux 3 points $\mathrm{T} 1, \mathrm{~T} 10$, T150 apparaissent sur la figure 8 où l'on y voit la bonne concordance entre le modèle complet et le modèle réduit identifié, avec toutefois un petit décrochement au voisinage de $t=0$ pour la température T150.

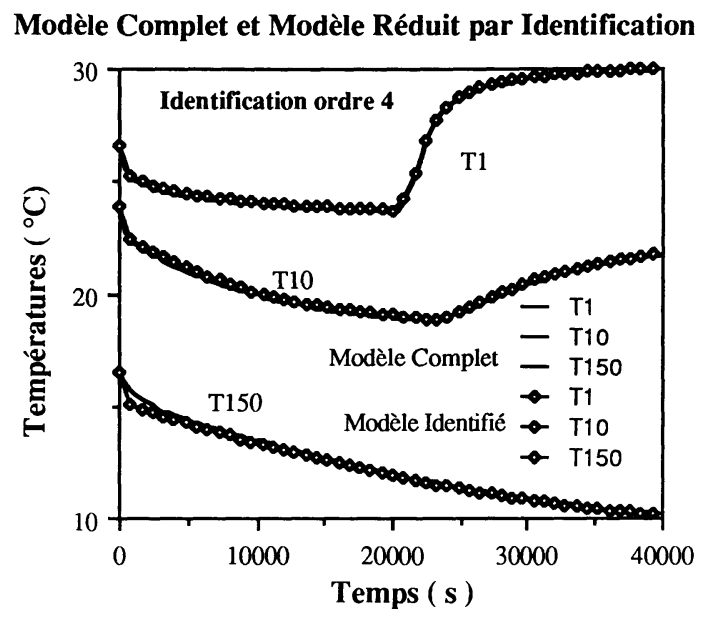

Fig. 8. - Comparaison du modèle complet et du modèle identifié d'ordre 4 avec une entrée fonction du temps suivant le tableau II.

[Comparison of the complete model and the identified one (order 4) with an input varying with the time according to table II.]

Les méthodes de Marshall, d'agrégation et d'Eitelberg ont été également testées sur cette simulation. Les résultats obtenus apparaissent dans les figures 9, 10 et 11. Comme précédemment, les méthodes modales donnent de bons résultats, bien que faisant

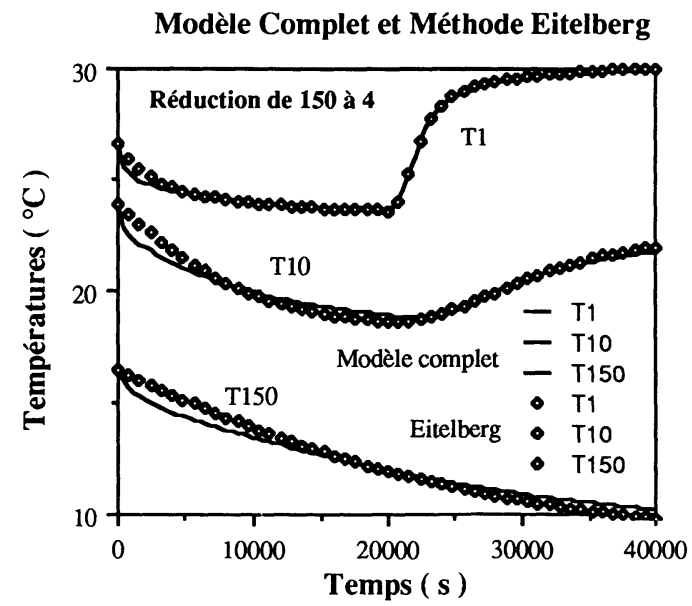

Fig. 9. - Comparaison du modèle complet et du modèle réduit d'Eitelberg d'ordre 4 avec une entrée fonction du temps suivant le tableau II.

[Comparison of the complete model and the Eitelberg reduced model (order 4) with an input varying with the time according table II.]
Modèle Complet et Méthode Marshall

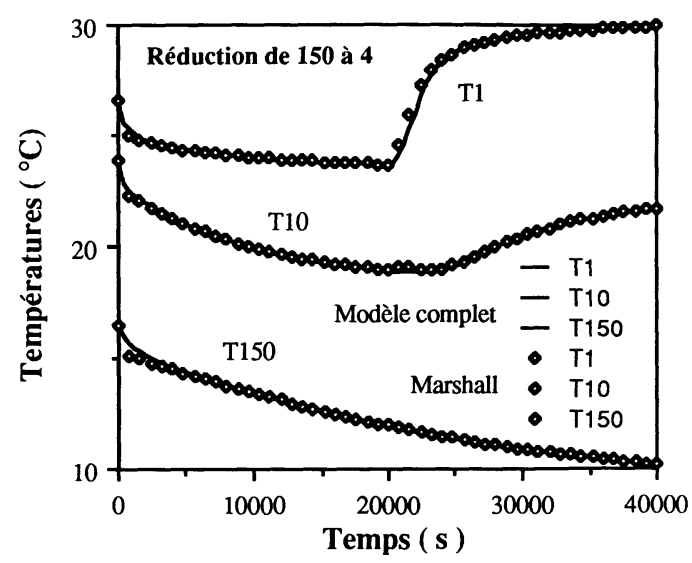

Fig. 10. - Comparaison du modèle complet et du modèle réduit de Marshall d'ordre 4 avec une entrée fonction du temps suivant le tableau II.

[Comparison of the complete model and Marshall reduced model (order 4) with an input varying with the time according to table II.]

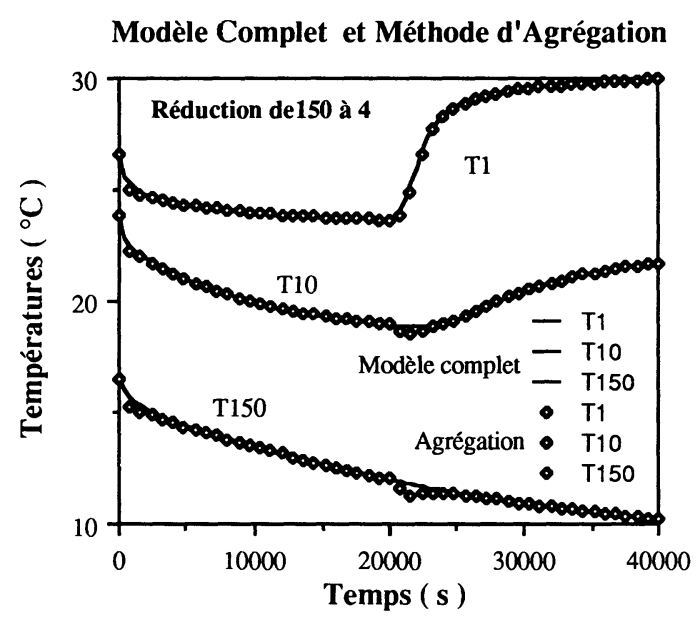

Fig. 11. - Comparaison du modèle complet et du modèle agrégé d'ordre 4 avec une entrée fonction du temps suivant le tableau II.

[Comparison of the complete model and aggregated model (order 4) with an input varying with the time according table II.]

apparaître de légers décrochements lors des variations brutales du vecteur d'entrée, ce qui n'est pas le cas pour la méthode d'Eitelberg.

5.4 CONSIDÉRATIONS NUMÉRIQUES. - Pour ce travail, les calculs ont été effectués avec une station de travail (microprocesseur Intel 80386, $25 \mathrm{MHz}$, coprocesseur Weitek). Au niveau des simulations numériques, les temps d'exécution correspondant aux différents modèles réduits sont semblables : tous inférieurs à $5 \mathrm{~s}$ pour la simulation du paragraphe 5.3.2 
par exemple alors que celui du modèle complet est de plusieurs minutes.

En ce qui concerne l'élaboration des matrices des modèles, les méthodes de Marshall, d'agrégation et d'Eitelberg nécessitent essentiellement des calculs de modes propres, d'inversion de matrice (ici d'ordre 150) et de produit matriciel. L'obtention des modèles ne dépasse alors pas $10 \mathrm{~min}$.

La procédure d'identification que nous avons mise au point est évidemment plus lourde car faisant appel aux techniques de P.N.L. (cf. Annexe) et le temps de calcul augmente rapidement avec l'ordre du modèle réduit : $6,30,50$ et $230 \mathrm{~min}$ respectivement pour identifier 1, 2, 3, 4 exponentielles; cette identification porte de plus ici sur un nombre élevé de températures : $150 \times 200=30000$. Par ailleurs, on peut envisager une amélioration de la procédure dans la mesure où les premières valeurs propres identifiées correspondent à celles de la matrice d'évolution : initialisation de la procédure d'optimisation d'après les plus grandes constantes de temps; néanmoins, cette technique suppose a priori un ordre minimal pour le modèle identifié.

Bien que l'obtention d'un modèle réduit nécessite la plupart du temps de l'espace mémoire et du temps de calcul, cet inconvénient ne constitue cependant pas un obstacle majeur : ces calculs préliminaires ne devant être fait qu'une seule fois peuvent être effectués sur un ordinateur adéquat, les modèles réduits pouvant ensuite être utilisés sur un ordinateur plus modeste.

\section{Conclusion.}

$\mathrm{Au}$ cours de cette étude, différentes formulations de la représentation d'état des systèmes linéaires ont été présentées, ainsi que leur répercussion au niveau du problème de la réduction. Cette approche nous a conduit à adopter une structure de modèle bien adaptée: la représentation d'état dans la base modale en faisant intervenir la dérivée temporelle $\mathrm{du}$ vecteur des entrées. Cette formulation permet de dissocier, au niveau des sorties, d'une part une composante statique et d'autre part une composante dynamique venant «corriger » le terme précédent. La composante statique assure de la conservation des régimes stationnaires, tandis que la méthode de réduction est appliquée sur la composante dynamique. Cette structure de modèle doit permettre une extension à la réduction des systèmes faiblement non linéaires.

Par rapport aux autres méthodes modales, l'originalité de notre approche est d'identifier les modes dominants du système et non pas de les sélectionner. Cette procédure permet essentiellement un amalgame naturel des modes et ainsi d'éviter la difficile sélection des modes dominants. Elle met également en évidence une matrice d'observation et la matrice d'entrée est ensuite déterminée par minimisation d'un écart entre les sorties des modèles complet et réduit. Nos recherches concernent actuellement la mise au point de méthodes d'affinement de ces matrices d'entrée et d'observation, en particulier en utilisant la notion de modes correcteurs introduite en [16].

Nous avons montré, sur un exemple de modélisation de plancher chauffant, comment on pouvait se satisfaire d'un modèle dynamique d'ordre 4 au lieu de 150. Dans le cadre de cette application, les 2 premières constantes de temps identifiées correspondent à celles du spectre de la matrice d'évolution : une méthodologie mettant en œuvre simultanément les méthodes modales classiques et notre procédure d'identification devrait être possible et fournir ainsi un outil de réduction particulièrement efficace. Ces résultats sont donc encourageants pour la réduction de modèle dont les applications devraient être nombreuses en diffusion thermique, compte tenu des facteurs de réduction très élevés pouvant être obtenus.

\section{Annexe 1.}

Notons :

$K_{i j}$ les termes de la matrice $\mathbf{K}$,

$k$ l'indice de discrétisation temporelle, $t(k)$ le temps correspondant,

$m$ la dimension du modèle réduit à identifier,

$Z_{j}=\exp \left(\lambda_{j}\right)$ où $\lambda_{j}$ est la $j$-ième valeur propre à identifier,

$f_{i}(u)$ la $i$-ième composante de $f(u)$,

$r$ le nombre de mesures par thermogramme, $q$ la dimension du vecteur de sortie.

A l'équation matricielle (14) correspond un ensemble de $q * r$ égalités de la forme :

$$
\hat{y}_{i}(k)-f_{i}[u(k)]=\sum_{j=1}^{m} K_{i j} Z_{j}^{t(k)} \text {. }
$$

En notant $\Delta y_{i}(k)$ l'écart entre les composantes numéro $i$ des vecteurs de sortie des modèles complet et réduit à l'instant $k$, introduisons le critère quadratique $C$ :

$$
C(m)=\sum_{i=1}^{q} C_{i} \quad \text { avec : } \quad C_{i}=\sum_{k=1}^{r}\left[\Delta y_{i}(k)\right]^{2} .(\mathrm{A} 2)
$$

Ce critère $C$ est fonction de la dimension $m$ du modèle à identifier (via le vecteur $Z$ ). On se propose d'estimer le paramètre $m$ en incrémentant la valeur de $m$ jusqu'à ce que :

$$
C(m+1) \approx C(m) .
$$

Pour une valeur déterminée de $m$, la minimisation du critère $C(m)$ permet le calcul du vecteur $Z$ (donc des modes dominants) et de la matrice $\mathbf{K}$ :

$$
[Z, \mathbf{K}](m)=\arg [\min C(m)] .
$$


Les équations (A3) et (A4) signifient que pour chaque valeur de $m$ il faudra résoudre un problème d'optimisation (non convexe) dont $[Z, \mathbf{K}]$ sont solutions. On doit $a$ priori remarquer que :

a) connaissant $Z$ de dimension $m$, chaque ligne de la matrice $K$ peut être déterminée de façon indépendante par la minimisation du critère $C_{i}$;

b) le domaine de résolution de $Z$ est tel que: $0<Z_{i}<1$ car la fonction exponentielle est positive et les valeurs propres sont réelles et négatives ;

c) si en (A1), $t^{\prime}(k)=t(k) / \alpha$ et $Z_{j}^{\prime}=Z_{j}^{\alpha}$, alors :

$$
Z_{j}^{t^{\prime}(k)}=Z_{j}^{t(k)} \text {. }
$$

Pour chaque valeur de $m$, nous avons mis en œuvre une méthode de descente opérant alternativement dans le sous-espace des $Z_{i}$, puis dans ceux des lignes de la matrice $\mathbf{K}$; mais d'après a), tout se passe comme si la fonctionnelle à minimiser n'était fonction que du vecteur $Z$. Les $q$ minimisations des $C_{i}$ reviennent alors à résoudre indépendamment $q$ systèmes linéaires que l'on peut regrouper sous la relation matricielle :

$$
\mathbf{W}^{\mathrm{T}} \mathbf{W K}^{\mathrm{T}}=\mathbf{W}^{\mathrm{T}} \mathbf{Y}
$$

avec :

$\mathbf{W}=\left[\begin{array}{ccc}Z_{1}^{t(1)} & \ldots & Z_{m}^{t(1)} \\ \ldots \ldots \ldots \ldots \ldots . \\ \ldots \ldots \ldots \ldots \ldots \ldots \\ Z_{1}^{t(r)} & \ldots & Z_{m}^{t(r)}\end{array}\right] \quad \mathbf{Y}=\left[\begin{array}{ccc}y_{1}(1) & \ldots & y_{q}(1) \\ y_{1}(2) & \ldots & y_{q}(2) \\ \vdots & \ldots & \vdots \\ y_{1}(r) & \ldots & y_{q}(r)\end{array}\right]$
Soit $\hat{Z}(m-1)$ la solution de (A4) pour une dimension $m-1$ de $Z$. Partant de cette valeur, on adjoint une composante supplémentaire $Z_{m}$ et l'on minimise le critère $C(m)$ tout d'abord par la méthode des dichotomies successives suivant cette nouvelle composante (entre 0 et 1 compte tenu de b)) en résolvant $\partial C(m) / \partial Z_{m}=0$. Soit $\bar{Z}(m)$ le vecteur ainsi obtenu. La solution finale $\hat{Z}(m)$ sera obtenue en appliquant un algorithme classique de PNL : la méthode des gradients conjugués [19]. Les relations suivantes permettent le calcul numérique du gradient du critère :

$$
\begin{gathered}
\partial C / \partial Z_{\mathrm{d}}=\sum_{i=1}^{q} \partial C_{i} / \partial Z_{\mathrm{d}} \\
\partial C_{i} / \partial Z_{\mathrm{d}}=\sum_{k=1}^{r} 2\left[\Delta y_{i}(k)\right] \partial y_{i}(k) / \partial Z_{\mathrm{d}} .
\end{gathered}
$$

En dérivant (A1) et (A6) il vient :

$$
\begin{aligned}
\partial y_{i}(k) / \partial Z_{\mathrm{d}}=\sum_{\ell=1}^{1} \partial K_{i \ell} / \partial Z_{\mathrm{d}} Z_{\ell}^{t(k)}+K_{i \mathrm{~d}} Z_{\mathrm{d}}^{t(k)-1} \\
\partial \mathbf{K}^{\mathrm{T}} / \partial Z_{\mathrm{d}}=\left(\mathbf{W}^{\mathrm{T}} \mathbf{W}\right)^{-1} \\
{\left[\partial \mathbf{W}^{\mathrm{T}} / \delta Z_{\mathrm{d}} \mathbf{Y}-\partial\left(\mathbf{W}^{\mathrm{T}} \mathbf{W}\right) / \partial Z_{\mathrm{d}} \mathbf{K}^{\mathrm{T}}\right] .(A}
\end{aligned}
$$

Remarquons enfin que l'utilisation de la propriété c) permet d'éviter de manipuler des exposants élevés et de "séparer" ainsi les composantes de $Z$.
[1] Decoster et al., A comparative study of Different Reduction Methods Journal A, vol. 17, $\mathrm{n}^{\circ} 3$ (1976).

[2] Marshall S., An approximate method for reducing the order of linear system, Control (1966) pp: 642-643.

[3] Fossard A., On a method for simplifying linear dynamic systems, IEEE Trans. Vol. AC-15 (1970).

[4] Michailesco, Approximation des Systèmes Complexes par des Modèles de Dimension Réduite, Thèse d'état Orsay 2/04/79.

[5] LITZ L., Order reduction of linear state space models via optimal approximation of the nondominant modes (1981) North-Holland Publishing Company, Large Scale System 2 (1981) 171-184.

[6] ShiH T. M., Skaladany J. T., An eigenvalue method for solving transient heat conduction problems, Numerical Heat Transfer 6 (1983) 409. 422.

[7] Sicard J., Bacot P., Neveu A., Analyse Modale des Echanges Thermiques dans le Bâtiment, Int. J. Heat Mass Transfer 28 (1985) 111-123.

[8] Lefebvre G., Bransier J., Neveu A., Simulation du comportement thermique d'un local par des méthodes numériques d'ordre réduit, Rev. Gén. Therm. 302 (1987) 106-114.

[9] Salgon J. J., Analyse Modale par discrétisation spatiale, Thèse Université Paris VI, 1987.

[10] SfAXI Y., Grignon M., Modélisation d'une structure thermique par un nombre réduit de paramètres, Rev. Gen. Therm. $\mathrm{n}^{\circ} 317$ (juin 1988).

[11] EITElberg E., Interactive Model Reduction by Minimizing the Weighted Equation Error, IFAC Symposium Toulouse.

[12] JaAfar T. B., Pasquetti R., Petit D., Model reduction for thermal diffusion. Int. J. for Num. Meth. Eng. 29 (1990) 599-617.

[13] Peube J. L. et al., Réduction des modèles de systèmes thermiques, Colloque des Systèmes et des Procédés 6 et 7 décembre 1988, CNRS Paris.

[14] Bertrand P., Duc G., Michailesco G., Recent developments in model reduction, RAIRO APII 19 (1985) 131-146.

[15] El KHOURY K., Formulation modale de problèmes de diffusion thermique avec transport (1989) Thèse Paris, Ecole des mines.

[16] Pasquetti R., Petit D., Analyse Modale d'un Processus de Diffusion Thermique: Identifica- 
tion par Thermographie Infra-Rouge, Int. J. Heat Mass Transfer Vol. 31 (1988).

[17] Eyкhоғf P., System Identification, Parameter and State Estimation (John Wiley \& Sons) 1974.

[18] Mérour P., La réduction de modèle en thermique. Application à l'étude d'un circuit intégré électronique, Thèse Poitiers (1986).

[19] Ciarlet P. G., Introduction à l'analyse numérique matricielle et à l'optimisation (Editions Masson, Paris) 1982.

[20] BARRAUD A., Réalisation minimale et approximation optimale des systèmes dynamiques linéaires invariants, Thèse Nantes (1975).

[21] Bistritz Y., Shaked U., Minimal Padé Model Reduction for multivariable systems, J. Dyn. Syst. Measur. Control. Vol. 106 (Dec. 1984). 